\begin{tabular}{|c|c|c|}
\hline Beitr. Ent. & Keltern & ISSN 0005-805X \\
\hline $\mathbf{5 6}(2006) 1$ & S. $5-22$ & 15.08 .2006 \\
\hline
\end{tabular}

\title{
Chronologische Tafel zur Geschichte des Deutschen Entomologischen Instituts 1886 - 2006
}

Et meminisse et vaticinari liceat.

Mit 2 Figuren

Holger H. Dathe

Summary

Essential events and stages of the history of German Entomological Institute are listed in chronological arrangement. Earlier reviews are actualized and critically completed. A continuation is planned using the internet-based "living review" mode [http://www.zalf.de/home_zalf/institute/dei/dei].

\section{Zusammenfassung}

In tabellarischer Form werden - nach Jahreszahlen angeordnet - die wesentlichen Ereignisse und Etappen der Entwicklung des Deutschen Entomologischen Instituts dargestellt. Frühere Übersichten werden auf den aktuellen Stand gebracht und kritisch ergänzt. Künftig ist eine ständige Aktualisierung im Living-ReviewModus vorgesehen [http://www.zalf.de/home_zalf/institute/dei/dei].

Key words

Deutsches Entomologisches Institut, history

\section{Einleitung}

Das Deutsche Entomologische Institut (DEI) vollendet im Juni 2006 sein 120-jähriges Bestehen, dem Referenz zu erweisen ist. Jubiläen sind wichtig, wenn sie mit dem Alter vor allem die erwiesene Lebenstüchtigkeit des Jubilars ins Bewusstsein rufen. Über eine altehrwürdige Einrichtung wie das DEI, das buchstäblich der Gründerzeit entstammt, ist naturgemäß bei verschiedenen Anlässen schon geschrieben worden (HoRN 1936, Korschefsky 1936, Sachtleben 1936, 1962, Fankhänel 1966, Ebert et al. 1986, Petersen 1986, Dathe \& Rohlfien 1994, Gaedike 1995 u. a.), so dass dem kaum etwas hinzugefügt werden muss. Andererseits werden immer wieder Daten und Fakten zur Historie des DEI angefragt, und dafür sollte eine sorgsam geprüfte Übersicht bereit sein. Für die hier aufbereitete chronologische Version wurden zahlreiche Originalquellen des DEI-Archivs geprüft, woraus sich diverse Korrekturen und Ergänzungen ergaben. Künftig sollen auch die neuen Möglichkeiten der Living Reviews genutzt werden. Die

\footnotetext{
${ }^{1}$ Erweiterte Fassung nach dem Eröffnungsvortrag zum SIEEC XIX in Müncheberg am 6.09.2005.
} 
nachfolgende Zeittafel wird in einer ständig zu aktualisierenden Version auf der Website des DEI erscheinen unter http:/www.zalf.de/home_zalf/institute/dei/dei/.

Es zeigt sich immer wieder, dass aktuelle Entwicklungen an dieser für Deutschland einzigartigen Institution von allgemeinerem Interesse sind, so dass sich die Fortschreibung der Chronik ohnehin empfiehlt. Nicht als Selbstbespiegelung, sondern mit ganz realem Bezug zu aktuellen Ereignissen und Anforderungen, die sich in der konzeptionellen Ausrichtung des Instituts wiederfinden müssen. Seine noch heute lesenswerten Kommentare und Glossen in den „Entomologischen Mitteilungen“, der Institutszeitschrift seit 1912, überschrieb Walther Horn „Et meminisse et vaticinari liceat" (,Es sei erlaubt, sich zu erinnern wie vorauszusagen"). Churchill soll es so gesagt haben: "Je weiter man zurückschaut, desto mehr

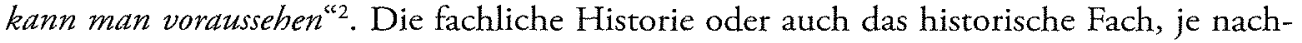
dem, ist ein unabdingbares Charakteristikum eines Forschungsmuseums, das sich mit der Dokumentation der Biodiversität in Schrift, Bild und Präparat zu befassen hat. Sie ist somit eine unverzichtbare Grundlage jeder weiteren Forschung und Erhaltung, von Theorie und Praxis im Umweltkontext, und insoweit sind unsere Aufgaben heute anspruchs- und verantwortungsvoller denn je. Bis zum Ruhestand von Dr. Klaus Rohlfien Ende 1999 verfügte das Institut über einen in der Auswertung von Archivalien ausgebildeten Experten, dessen Werk bis heute in Bibliothek, Publikationswesen und Öffentlichkeitsarbeit nachwirkt, aber die hinterlassene Lücke wird zunehmend deutlicher. Eine gute Tradition und einen guten Ruf, die nur in Jahrzehnten aufgebaut werden können und die uns im Falle des Deutschen Entomologischen Instituts mit den Leistungen der alten Entomologen aufgegeben wurden, gilt es sorgsam zu pflegen, um sie für die Zeitaufgaben zu nutzen. In der Sache wollen wir es mit Gustav Mahler halten:

Tradition ist nicht die Verehrung der Asche, sondern die Anbetung des Feuers.

\section{Chronologische Tafel}

1886 Gründung des „Deutschen Entomologischen National-Museums“ (ENM) durch Vereinbarung zwischen Dr. Gustav Kraatz (1831-1909; Köppen 1967) und dem Direktorium des Märkischen Provinzial-Museums der Stadt Berlin. Es wird verabredet, im geplanten Neubau des Provinzialmuseums zwei bis drei Zimmer mittlerer Größe für das ENM vorzusehen. Vorerst werden die Bestände im städtischen Sparkassengebäude in der Zimmerstraße (Berlin-Mitte) untergebracht.

Bereits 1880 hatte Kraatz in der Deutschen Entomologischen Zeitschrift seine Sammlung europäischer und exotischer Käfer sowie seine an wertvollen Werken über Insekten aus allen Ordnungen reiche entomologische Bibliothek einem zu schaffenden Deutschen Entomologischen National-Museum übereignet.

Neben Gustav Kraatz haben durch Übereignung ihrer Kollektionen an ein ENM als „Urstifter" beigetragen:

Lucas von Heyden (1838-1915), Frankfurt am Main - Kollektion paläarktischer Coleoptera

Karl Wilhelm Letzner (1812-1889), Breslau - Kollektion europäischer Coleoptera

Georg Metzler (1863-1880), Frankfurt am Main - Kollektion Coleoptera: Melolonthinae

William Henry Rolph (1847-1883), Berlin - Kollektion marokkanischer Coleoptera

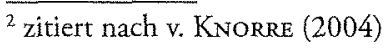


August John Stern (1822-1879), Frankfurt am Main - Kollektion Coleoptera Johann Carl Stern (1823-1886), Frankfurt am Main - Kollektion Coleoptera (v. a. Deutschland)

Mit der genannten Vereinbarung erreichen die seit 1870 verfolgten Bemühungen Kraatz' um das ENM ihr Ziel. Die Rechtsform ist die private gemeinnützige wissenschaftliche Stiftung, die als Schenkung an die Stadt Berlin gehen soll (Stiftungsurkunde vom 7.06.1886).

„Das Deutsche Entomologische National-Museum will Sammlungen und Bibliotheken, besonders deutscher Entomologen, vereinen, verwalten und allen Entomologen zugänglich machen, um so ein rein entomologisch verwaltetes Zentrum der deutschen Entomologie zu schaffen". (KRAatz 1880)

1887 Der König von Preußen erteilt die Allerhöchste Genehmigung zur Annahme dieser schenkungsweisen Zuwendung (28.04.).

1889 Erste Begegnung zwischen Kraatz und dem 18-jährigen Walther Horn (1871-1939).

1903 Das Märkische Provinzialmuseum wird verabredungswidrig ohne zoologische Abteilung eröffnet. Kraatz ändert daraufhin die Stiftungsbestimmungen zugunsten einer eigenständigen „Dr. Kraatz’schen Stiftung von 1903“. Er kauft das Haus Thomasiusstraße 21 in Berlin-Moabit und nutzt ab 1904 eine der Etagen für das Museum.

1906 Die Kraatz-Bibliothek, die der Berliner Buchhändler Ulrich aufstellt, umfasst 1403 Bände Käferliteratur, 628 Bände über Schmetterlinge, 635 Bände zu übrigen Insekten sowie 58 meist vollständige Zeitschriftenreihen; dazu kommen über 7000 Sonderdrucke. Der Leihverkehr ist bereits umfangreich.

1907 In einem testamentarischen Nachtrag ernennt Kraatz den Entomologen Dr. med. Walther Horn zum Direktor des Deutschen Entomologischen National-Museums auf Lebenszeit.

1909 Die Königliche Kommission zur Aufteilung der Domäne Dahlem verkauft eine Parzelle an Prof. Dr. Gustav Kraatz (20.01.). Kraatz stirbt am 1. November. Er hinterlässt der Stiftung das Baugrundstück und ein Vermögen von 853000 Goldmark.

„Die Verdienste von Prof. Kraatz für das Museum liegen in dessen Gründung, deren Notwendigkeit er erkannte und wozu er durch seine Sammlungen und Bibliothek den Grundstock gelegt hat. Darüber hinaus hat er sich durch seine vielseitige wissenschaftliche Arbeit über Coleoptera, über Faunistik, über naturwissenschaftliche Literatur einen international anerkannten Namen gemacht." (EBERT et al. 1986)

1910 Herausgabe einer ersten eigenen Zeitschrift („Deutsche Entomologische National-Bibliothek") und Beginn des internationalen Schriftentausches.

„Mit der Zeitschrift wird die Absicht verfolgt, aus der bereits bestehenden Bibliothek eine große "Deutsche Entomologische National-Bibliothek" zu entwickeln, indem alle durch Kauf, im Schriftentausch, als Rezensionsexemplar oder als Geschenk eingehenden Publikationen ebenso wie der bisherige Bestand den Abonnenten der Zeitschrift und anderen Entomologen leihweise zur Verfügung stehen." (EBERT et al. 1986)

Beginn des Instituts-Neubaus in Berlin-Dahlem, Gosslerstraße 20. Architekt: Heinrich Straumer (1876-1937), der unter vielem anderen auch den Berliner Funkturm erbaute. 
1911 Erbschaftsannahme durch die Stadt Berlin, verbunden mit der Namensänderung in "Deutsches Entomologisches Museum" (DEM). Der landesherrlichen Anerkennung (11.12.) ging eine Auseinandersetzung mit dem Direktor des Kgl. Zoologischen Museums der Berliner Universität, Prof. Dr. A. Brauer, um die Eigenständigkeit der Kraatz'schen Stiftung voraus (Horn 1912, 1922).

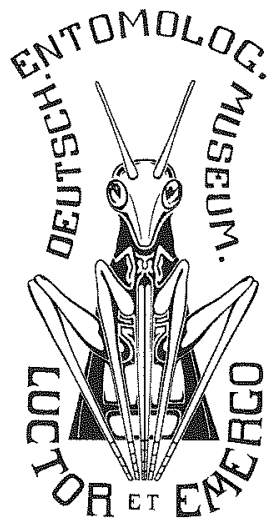

Fig. 1. Signet des Deutschen Entomologischen Museums. In der existenziellen Bedrängnis ob der Eigenständigkeit der Kraatz'schen Stiftung entsteht 1911 auch das Signet des Deutschen Entomologischen Museums mit der Heuschrecke und dem Wahlspruch "Luctor et emergo", das fortan als „ex libris" der Bibliothek wie auch auf den Veröffentlichungen und Briefköpfen geführt wird. Die Heuschrecke, der man ganz im Sinne des Jugendstils sozusagen „auf gleicher Augenhöhe" ins Gesicht schaut, soll - wie durch Sachtleben überliefert ist - die "Weltmacht Insekten" symbolisieren. Der Wahlspruch ist die Devise der Zeeländer im Kampf gegen die Sturmfluten der Nordsee. Schöpfer des Signets sind W. Horn, der Architekt H. Straumer, der Assistent K. H. Wagner und der Kunstmaler E. Bischoff-Culm.

1912 Einweihung des Museumsneubaus in Berlin-Dahlem (2.11.). Neben Walther Horn als Direktor arbeiten am DEM der Kustos Berthold Sigismund Schenkling (seit 1904), der Assistent Karl Hans Wagner (seit 1911) und eine Schreibkraft.

Um der Systematik breiteren Raum in den Veröffentlichungen zu geben, ediert das DEM (formal in Gestalt des Vereins zur Föderung des "Deutschen Entomologischen Museums") anstelle der "Deutschen Entomologischen National-Bibliothek" (24 Hefte in 2 Bänden, 1910 und 1911) eine Monatsschrift mit dem Titel "Entomologische Mitteilungen" (1912 bis 1929). Für umfangreichere Arbeiten wird eine Schriftenreihe „Supplementa Entomologica" (1913 bis 1929) ins Leben gerufen. Horn begründet dies u. a. mit der Notwendigkeit, „einen breiteren Raum für die Systematik in den Veröffentlichungen des Museums bereit zu stellen". Die Bibliothek gewinnt damit eine bessere Basis für den Schriftentausch. Sie tritt der „Auskunftsstelle der deutschen Bibliotheken“ bei und bedient die Fernleihe.

1914-1918 Der Erste Weltkrieg unterbricht den internationalen Austausch, ab 1915 gibt es keinen Personaletat mehr. Horn selbst meldet sich gleich zu Anfang des Krieges und geht 1915 als Feldarzt an die Ostfront. Er findet nach vier Jahren das Museum „in seinen Grundfesten ruiniert" vor (HoRN 1922). Die Geld- und Anlagenentwertung nach dem Krieg bringt große finanzielle Schwierigkeiten. Horn versucht, eine „wirkliche 
Übernahme“ durch die Stadt Berlin oder - über das Reichsernährungsministerium - einen Anschluss an die Biologische Reichsanstalt für Land- und Forstwirtschaft zu erreichen.

1920 Magistratsbeschluss vom 18.11. zur Namensänderung in „Deutsches Entomologisches Institut" (DEI) auf Vorschlag W. Horns, „da das Museum tatsächlich längst durch seine ganze Entwicklung ein internationales wissenschaftliches Forschungsinstitut geworden ist" (Horn an den Vorsitzenden des Kuratoriums, 20.10.). Die Bezeichnung "Museum“ wird als Hindernis für die gewünschte Übernahme durch Reichsbehörden betrachtet. Bemühungen zur Unterstellung unter das Reichsernährungsministerium scheitern, aber es entsteht eine enge Beziehung zur Biologischen Reichsanstalt in Dahlem, die fortan erhalten und gepflegt wird.

Bestände: 150000 Insekten, darunter6 000Typen; aufgestellt in 142 Schränken; Alkoholmaterial in 343 Standgefäßen. Bibliothek: 13000 Bände, 18000 Separata; 119 Zeitschriften im Tausch gegen die beiden eigenen Periodika; rund 1600 Entomologen-Portraits und zahlreiche weitere Dokumente.

1922 Nach schwierigen Verhandlungen wird das Institut zum 1.10. der Kaiser-WilhelmGesellschaft zur Förderung der Wissenschaften (KWG) angegliedert; Bestände, Liegenschaften und Restkapital gehen an die KWG über, die Kraatz'sche Stiftung erlischt. Horn wird als Direktor übernommen, der Kustos Schenkling (1865-1946) wird Stadtangestellter. Wissenschaftspolitisch ist die Bindung an die KWG eine vertretbare Lösung, finanziell jedoch praktisch ohne Auswirkung.

Leitgedanken der KWG, die mit den grundlegenden Zielstellungen des Deutschen Entomologischen Instituts übereinstimmten:

1. die Förderung der wissenschaftlichen Arbeit unabhängig von den Aufgaben der Lehre an den Universitäten,

2. die Förderung von Spezialgebieten, die für die Lehre nicht in vollem Maße nutzbar gemacht werden können,

3. die Förderung von wissenschaftlichen Instituten, die bestimmten Industriezweigen kontinuierliche Anregungen geben sollten,

4. die stärkere Betonung des nationalen Gedankens in der Wissenschaft.

Aufgaben des DEI, „\$1. Die Kaiser-Wilhelm-Gesellschaft zur Förderung der Wissenschaften übernimmt das Deutsche Entomologische Museum der Stadt Berlin (Kraatz'sche-Stiftung) zu dem Zwecke, wissenschaftliche Forschungen auf dem Gebiete der Entomologie zu fördern."

Am 1.11. tritt Frau Christine Doering (geb. 1892) ihren Dienst als Bibliothekarin des DEI an, den sie bis 1958, nahezu 36 Jahre lang, als "Seele der Bibliothek" versieht (Fig. 2).

1925 Das Reichsernährungsministerium übernimmt einen Teil des Institutsetats. Das Ministerium verlangt dafür die Einrichtung eines „Auskunfts- und Vermittlungsdienstes“ für entomologische Fragen. Zu diesem Zweck werden die bibliographischen Arbeiten zur Hauptaufgabe entwickelt („Index“, „Ringkartei"), und es werden die biologischen Sammlungen ausgebaut.

1926 Organisation der ersten „Wanderversammlung Deutscher Entomologen " in Halle (Saale) durch Horn und Mitarbeiter. 


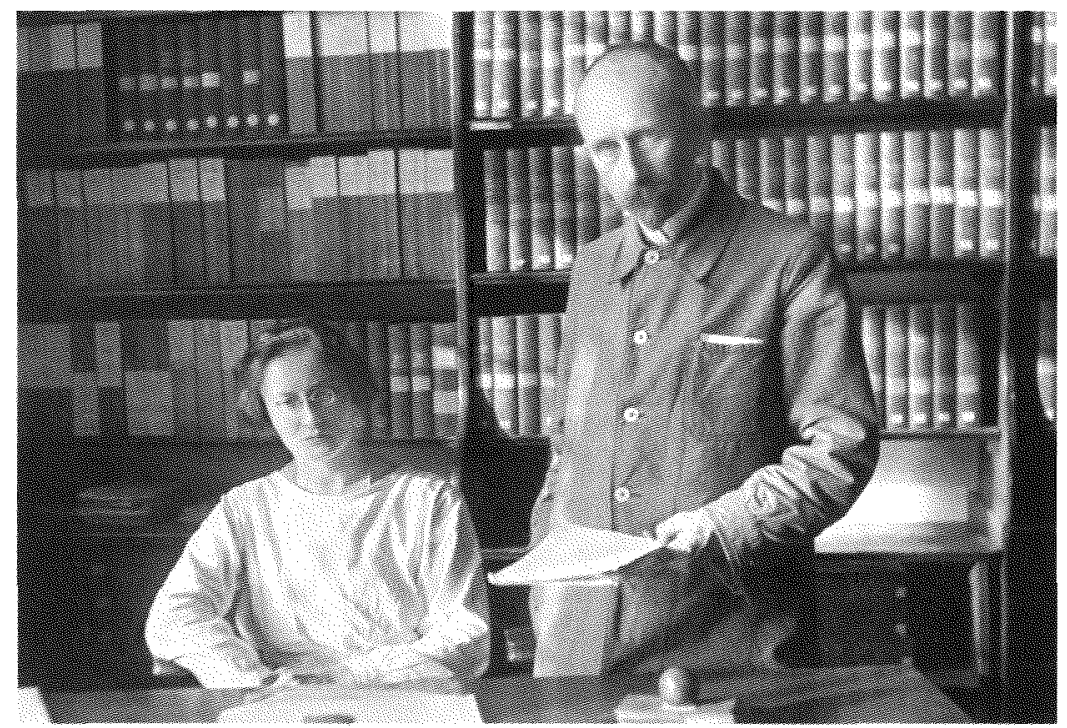

Fig. 2: Christine Doering, die Bibliothekarin, und Walther Horn (1927). Sie wohnt im Institutsgebäude und geht 1943 mit nach Blücherhof. Durch ihr Gedächtnis und ihre Sprachkenntnisse, insbesondere aber ihre Fähigkeit, bei der bibliothekarischen Aufarbeitung der eingehenden Schriften auch wesentliche Inhalte mit zu erfassen und reproduzierbar zu behalten, wird sie zur allerseits geachteten Autorität. Das Institut verdankt ihr in hohem Maße seinen Ordnungszustand und damit seine Funktionsfähigkeit. Aufn.: DEIArchiv (Psig. 854).

1928 Erscheinen des „Index Litteraturae Entomologicae“, der Bibliographie der entomologischen Weltliteratur bis 1863, womit der bibliographische Anschluss an den Zoological Record hergestellt wird. Das DEI erlangt mit dieser überragenden Leistung Weltgeltung (Horn \& Schenkling 1928, Gaedike \& Smetana 1978, 1984).

Darauf aufbauend, schlägt Horn auf dem IV. Internationalen Entomologen-Kongress in Ithaca die Gründung eines „Entomologischen Instituts für internationalen Dienst" in Zürich vor, dessen Grundstock das DEI bilden soll. Es wird ein Komitee eingerichtet, dass sich mit diesem Plan befassen soll, aber der Versuch bleibt letztlich erfolglos. Hemmnisse sind die internationale politische Lage sowie der Stand der Informationstechnik und der Wissenschaftsorganisation.

1932 Die Bibliothek umfasst 10858 Zeitschriftenbände, 6827 Einzelwerke und 55000 Sonderdrucke. Horn apostrophiert die B15 als die „größte entomologische Spezialbibliothek Europas".

1933 Erneute Bestrebungen, das DEI aufzulösen und Sammlungen und Bibliothek abzugeben, diesmal an das Museum Alexander Koenig nach Bonn, können von Horn abgewendet werden. Nach langwierigen Verhandlungen zwischen dem Reichs-Landwirtschaftsministerium und der Kaiser-Wilhelm-Gesellschaft wird am 18.12. eine Arbeitsgemeinschaft der Biologischen Reichsanstalt für Land- und Forstwirtschaft (BRA) mit dem DEI geschlossen. Zweck: Förderung der systematischen und morphologischen Forschung als Grundlage für die Arbeiten der Biologischen Reichsanstalt auf dem Gebiet der angewandten Entomologie.

Schwerpunkt ist die gemeinsame Herausgabe einer entomologischen Zeitschrift mit drei Abteilungen als eigene Publikationsorgane und Basis für den Schriftentausch: 
(1) „Arbeiten über physiologische und angewandte Entomologie" (erschienen 1934 bis 1944),

(2) „Arbeiten über morphologische und taxonomische Entomologie“ (1934 bis 1944),

(3) „Entomologische Beihefte" (1934 bis 1943).

1934 Als Vertreter der Biologischen Reichsanstalt für die gemeinsamen wissenschaftlichen Aufgaben in der Arbeitsgemeinschaft wird der Regierungsrat Dr. Hans Sachtleben (18931967, Leiter des Forstzoologischen Laboratoriums der BRA) in das DEI abgeordnet und Stellvertreter von W. Horn.

Sachtleben hat neben den Arbeiten, die ihm laut Vertrag oblagen, seine Aktivitäten allmählich immer mehr auf die traditionellen Aufgaben des DEI gerichtet, zunächst als Redakteur der Zeitschriften, mit denen das Institut bis in die ersten Kriegsjahre hinein international auf sich aufmerksam machte (PETERSEN 1986).

1935 Erscheinen der "Collectiones entomologicae" (Verbleib entomologischer Sammlungen, Geschichte der Entomologie und Museologie), bis 1937 in drei Folgen (Horn \& KaHle 1935-1937).

Der "Sammlungsverbleib“ ist ob seiner internationalen Bedeutung die wichtigste und eindrucksvollste Leistung dieser Periode. „Es hätte einer größeren und international bedeutenderen Institution, z. B. dem Britischen Museum (N. H.) in London, sehr wohl angestanden, ein solches Werk zu erarbeiten. Man hatte dort alle Voraussetzungen, bis auf eine - den Mann, der so etwas als machbar erkannte, es anpackte und in kürzester Zeit realisierte." (PETERSEN 1986). - Aktualisierte Neuauflage in 2 Bänden: HoRN et al. 1990; Ergänzungen und Berichtigungen: GAEDIKE 1995.

1936 In seinem Artikel zum 50-jährigen Bestehen des DEI beschreibt R. Korschefsky die vergangenen Jahrzehnte als ständigen Kampf um die Existenz des Instituts, und er hebt die "geniale, umsichtige und kampfesmutige Leitung" durch Walther Horn hervor. Dem „völligen Einsatz seiner Person ist es zu verdanken, dass wir in dem Deutschen Entomologischen Institut eine Musterstätte ersten Ranges von internationaler Bedeutung haben" (KoRschefsky 1936). In der Tat ist Horn der geistige Schöpfer und Gestalter des DEI mit nachhaltiger Wirkung.

Die Insektensammlungen sind in 183 einheitlichen Schränken mit ca. 10000 Glaskästen aufgestellt. Sie enthält rund 25000 Typen und historische Exemplare. Alles vorhandene Insektenmaterial ist mit Hilfe von Kartothekkarten in kürzester Zeit zu finden. „Die Bibliothek ist die anerkannt größte Fachbibliothek auf dem Kontinent und umfasst 927 Zeitschriften mit entomologischem Inhalt, oft mit 100 Bänden und mehr pro Zeitschrif, sowie 8000 Nummern von Einzelwerken und Separatabänden. In über 400 Mappen sind außerdem lose Separata - etwa 65000 Stück - aufbewahrt. Eine besondere Rolle spielt die bibliographische Kartothek der gesamten entomologischen Literatur der Welt ab 1864. Sie ist als Fortsetzung des ,Index Litteraturae Entomologicae I' gedacht, mit dem Ziel einer späteren Veröffentlichung. In 264 Kästen sind bis heute bereits über 208000 Arbeiten registriert. (Die Gesamtzahl der entomologischen Arbeiten wird auf über 300000 geschätzt)." (KoRschefsKr 1936)

1938 1.10. Dr. Willi Hennig (1913-1976), der bereits seit 1937 als Stipendiat am Hause tätig ist, wird als wissenschaftlicher Assistent eingestellt.

1939 Dr. W. Horn stirbt am 10. Juli. Er hinterlässt ein Institut mit wertvollem, geordnetem Sammlungsbestand, mit einer umfassenden Fachbibliothek mit anspruchsvollen bibliographischen Vorhaben, mit einem Archiv und diversen Spezialkarteien für Auskünfte. 
Am DEI arbeiten 8 Entomologen. Im Schriftentausch werden 685 Zeitschriften bezogen. Als Nachfolger tritt Dr. H. Sachtleben zunächst kommissarisch ein, ab 1943 als Direktor.

Mit Kriegsbeginn wird W. Hennig zur Infanterie eingezogen. Nach einer Verwundung 1942 wird er als Entomologe bei der Malariabekämpfung eingesetzt.

1943 Kriegsbedingte Auslagerung des Instituts nach Schloss Blücherhof, Gemeinde Lutgendorf bei Vollrathsruhe (Kreis Waren/Müritz) in Mecklenburg.

Transport in 22 Eisenbahnwaggons ab 22.07., Abschluss 25.08. Sachtleben und Frau, die Sekretärin, die Bibliothekarin und eine Bibliothekshilfe ziehen mit um. Der Schriftentausch ist zusammengebrochen, aber der entomologische Auskunftsdienst zum Pflanzenschutz etc. wird umgehend wieder aufgenommen. Beim Institut verbleibt auch - mit Sachtlebens Hilfe mehrfach vom Militärdienst zurückgestellt - der Coccinelliden-Spezialist Richard Korschefsky (geb. 1902, seit 1927 Präparator und Technischer Inspektor am DEI), bis er noch Ende März 1945 eingezogen wird. Er stirbt 1946 in sowjetischer Kriegsgefangenschaft. - Mit persönlichem Einsatz verhindert Sachtleben Plünderungen bei Kriegshandlungen und nach Kriegsende.

1945 Nach dem Ende des Zweiten Weltkriegs kann die Kaiser-Wilhelm-Gesellschaft keine Mittel für den weiteren Betrieb des Instituts bereitstellen, sie kämpft um ihr eigenes Überleben. Sachtleben versucht, das Institut entweder bei der KWG oder bei der Biologischen Reichsanstalt anzugliedern. Das Berliner Museum für Naturkunde äußert sein Interesse an der Übernahme des DEI, allerdings ohne Personal. Prof. Dr. Hans Bischoff, Direktor des Zoologischen Museums, verhandelt darüber - an Sachtleben vorbei - mit der sowjetischen Militäradministration.

W. Hennig kehrt nach Berlin zurück, übernimmt aber zum Jahresende (1.12.) die Vertretung seines Doktorvaters Prof. Paul Buchner im Lehrfach Zoologie am Zoologischen Institut der Universität Leipzig (bis 31.03.1947).

1946 Sachtleben verwahrt sich mit Erfolg, und zum 1.01. wird das Deutsche Entomologische Institut der Biologischen Zentralanstalt für Land- und Forstwirtschaft in Berlin-Dahlem angeschlossen.

Am 25.12. teilt der Präsident der Deutschen Zentralverwaltung für Land- und Forstwirtschaft in der sowjetischen Besatzungszone brieflich mit: „Die Biologische Reichsanstalt wird mit allen ihren Zweig- und Außenstellen innerhalb der sowjetischen Zone mit Wirkung vom 1. Januar 1946 von der Deutschen Verwaltung für Land- und Forstwirtschaft in der sowjetischen Besatzungszone übernommen. Der Anstalt angegliedert werden das Deutsche Entomologische Institut, z. Zt. Blücherhof, Post Vollrathsruhe/Mecklenburg, und die staatlich anerkannte Vogelschutzwarte Seebach, Kreis Langensalza."

1947 Vom Präsidenten der Biologischen Zentralanstalt, Prof. Schlumberger, unterstützte Bemühungen um die Rückverlegung nach Berlin scheitern vorerst, denn das Dahlemer Institutsgebäude ist von der amerikanischen Militäradministration beschlagnahmt und eine Räumung nicht absehbar.

W. Hennig kehrt am 1.04. zum DEI zurück und übernimmt vor allem die Vermittlung zwischen dem Institut im Schloss Blücherhof und der Biologischen Zentralanstalt.

1949 Die politischen Gegebenheiten in der Viersektorenstadt Berlin wirken sich aus, das Institut muss im sowjetischen Besatzungsbereich verbleiben. Es wird mit Verfügung vom 17.09. der Hauptverwaltung Land- und Forstwirtschaft der Deutschen Wirt- 
schaftskommission für die sowjetische Besatzungszone (ab Oktober Ministerium für Land- und Forstwirtschaft der DDR) aus der Biologischen Zentralanstalt in BerlinDahlem (amerikanischer Sektor) herausgelöst und ihr direkt unterstellt.

Das DEI soll künftig nicht nur systematisch und bibliographisch arbeiten, sondern auch Grundlagenforschung auf dem Gebiet der angewandten Entomologie betreiben. Direktor bleibt Prof. Sachtleben, Stellvertreter wird Dr. Hennig. Der Stellenplan wird aufgestockt.

1950 6.02. bis 23.05. Rückführung des Deutschen Entomologischen Instituts nach Berlin, allerdings nicht in sein Stammhaus, sondern in den sowjetischen Sektor nach Friedrichshagen. Die Villa in der Waldowstraße 1 ist nur eine Notlösung und insbesondere für die neuen Aufgaben der Angewandten Entomologie völlig unzureichend. - Das Institutsgebäude in Dahlem beziehen Einrichtungen der Freien Universität.

Erscheinen der „Grundzüge einer Theorie der Phylogenetischen Systematik“ von Willi Hennig.

Hennig habilitiert sich an der Brandenburgischen Landeshochschule Potsdam für das Fach Zoologie und erhält ebendort eine apl. Professur (10.10.).

1951 10.01. Der Ministerrat der DDR beschließt die Gründung einer Deutschen Akademie der Landwirtschaftswissenschaften (DAL). Eine Anlage nennt auch das ihr zu unterstellende Deutsche Entomologische Institut in Berlin-Friedrichshagen. - Erstes Heft der neuen Zeitschrift „Beiträge zur Entomologie" (BE) erscheint. Damit wird erneut der Schriftentausch zum devisenfreien Erwerb internationaler Fachliteratur aufgenommen.

$1952 \mathrm{Ab}$ 1.01. fachliche und verwaltungsmäßige Übernahme in die Deutsche Akademie der Landwirtschaftswissenschaften zu Berlin.

H. Sachtleben zur Aufgabenstellung: „Als Verbindungsinstitut zwischen der systematischen und angewandten Entomologie hat das Deutsche Entomologische Institut die Aufgabe, die auf den Gebieten der angewandten Entomologie und des Pflanzenschutzes arbeitenden Stellen und Wissenschaftler in allen Fragen der systematisch-morphologischen Entomologie und der entomologischen Bibliographie zu unterstützen." (Interessanterweise nicht genannt wird die neue Aufgabe „Angewandte Entomologie ${ }^{\star}$.) - 3 Abteilungen:

Abteilung für allgemeine und bibliographische Entomologie (Leitung: Prof. Dr. Hans Sachtleben, bis 1962)

1. Bestimmungs- und Auskunftstätigkeit, 2. Bestandsaufbau, -erschließung und -vermittlung der Entomologischen Fachbibliothek, 3. bibliographische Arbeiten an der Kartothek der entomologischen Weltliteratur, 4. redaktionelle Bearbeitung der Institutszeitschrift „Beiträge zur Entomologie“, 5. Arbeiten in den wissenschaftlichen Sammlungen, 6. Forschungsarbeiten des Institutsdirektors über Systematik und Ökologie parasitischer Hymenopteren.

Abreilung für systematische Entomologie (Leitung: Prof. Dr. Willi Hennig, bis 1961)

Untersuchungen der Grundlagen bei der Erkennung und Unterscheidung der Insekten, insbesondere der land-, forst- und vorratswirtschaftlich, technisch und medizinisch-hygienisch wichtigen Formen in allen Lebensstadien und in ihren Schadbildern. - Grundlegende Arbeiten von Prof. Dr. Willi Hennig: dreibändiges Werk über „Die Larvenformen der Dipteren“, weitere theoretische Arbeiten zu seiner Theorie der Phylogenetischen Systematik, umfassende Bearbeitungen der Muscidae und Anthomyidae für Lindner, „Die Fliegen der palaearktischen Region“. Weitere taxonomische Arbeiten unter Erweiterung des Mitarbeiterstabes vor allem auf Coleoptera (Dr. Johann Machatschke), Microlepidoptera (Dr. Günther Petersen, Dr. Gerrit Friese) und parasitische Hymenopteren (Dr. Eberhard Königsmann). 
Abteilung für angewandte Entomologie (Leitung: Prof. Dr. Walter Tomaszewski, bis 1955) Diagnostische und ökologische Untersuchungen wichtiger Vorrats- und Materialschädlinge (Prof. Dr. W Tomaszewski). Biozönotische Grundlagen der Forstentomologie, Probleme der Bionomic, des Massenwechsels, der Prognose und der Schadwirkung (Dr. Wolfgang Schwenke).

An dieser Ziel- und Aufgabenstellung ändert sich bis zum Jahre 1961 nichts Wesentliches (DALJahrbücher).

1955 Die ,Abteilung für angewandte Entomologie "wird nach dem Tode von Prof. Tomaszewski (1903-1955) in „Abteilung für ökologische und biozönologische Entomologie" umbenannt. Als Leiter folgt ihm Dr. Wolfgang Schwenke (1921-2006); er bearbeitet jedoch nicht mehr Vorratsschädlinge; statt dessen wird Grundlagenforschung zur biologischen Bekämpfung, insbesondere der Massenwechsel bei wirtschaftlich wichtigen Insektenarten, betrieben.

Allerdings sind die räumlichen Voraussetzungen für entsprechende experimentelle Arbeiten denkbar schlecht. Die Raumsituation wird durch Wassereinbrücheverschärft, so dass Neubaupläne Chancen bekommen. Ein erstes Projekt wird von der DAL-Leitung abgelehnt (1958), ein weiteres (1959) vom Berliner Stadtarchitekten.

Beginn der bibliographischen Arbeiten zur Serie II des „Index Litteraturae Entomologicae“ für den Zeitraum 1864-1900 auf der Grundlage der bibliographischen Kartothek.

Der Präsident der DAL, Prof. Dr. Dr. h. c. mult. Hans Stubbe, nannte das Vorhaben „eine der Akademie wïrdige Aufgabe". Entsprechend wurde eine "Arbeitsgruppe Bibliographie" gebildet und mit 6 Personalstellen gefördert; Leiter: Dr. Walter Derksen, Mitarbeit: Dr. U. GöllnerScheiding und vier technische Angestelle.

1959 W. Schwenke verlässt das DEI und geht an die LMU München. Auf Wunsch von H. Sachtleben übernimmt Dr. Günther Petersen (geb. 1924) „treuhänderisch“ die ökologische Abteilung. Sachtleben bemüht sich im Einvernehmen mit der AkademieLeitung um den renommierten Entomo-Ökologen Prof. Dr. Hans Joachim Müller (Quedlinburg) für die Funktion, während W. Hennig dafür eintritt, die angewandte Arbeitsrichtung aufzugeben und nach Kleinmachnow zu verlagern. Die Unsicherheiten um einen Neubau und die intern in Frage gestellte Perspektive ökologischer Forschung am DEI führen zur Absage von H. J. Müller (Petersen 1986).

1960 Beginn der Drucklegung des „Index Litteraturae Entomologicae, Serie II“; abgeschlossen 1963. In der Institutszeitschrift „Beiträge zur Entomologie“ Start einer neuen Publikationsserie „Beiträge zur Insektenfauna der DDR“, die die vollständige Inventarisierung der Insekten des Gebiets zum Ziel hat.

1961 Albanien-Expedition des Instituts; G. Friese, E. Königsmann und Joachim Schulze sammeln rund 42000 Tiere aus nahezu allen Ordnungen. Die „Ergebnisse der AlbanienExpedition 1961 des Deutschen Entomologischen Instituts" werden nachfolgend in einer Publikationsserie der "Beiträge zur Entomologie“ veröffentlicht (bisher 101 Arbeiten). (Friese \& KönIgSMANN 1962)

W. Hennig, der in Berlin-Steglitz wohnt, muss als West-Berliner das DEI wenige Wochen nach dem Mauerbau verlassen (26.09.). Die Leitung der Abteilung für systematische Entomologie wird Dr. Günther Petersen übertragen. 
1962 G. Petersen übergibt die Leitung der ökologischen Abteilung zum 1.02. an Dr. Heinz Fankhänel (1927-1995), der sie bis 1970 mit dem neuen Namen „Abteilung für Ökologie der Insekten und biologische Schädlingsbekämpfung" führt. Wesentlicher Schwerpunkt der Arbeiten ist die biologische Schädlingsbekämpfung (Einsatz von Bakterienpräparaten, Trichogramma u. a.). Dr. Fankhänel wird gleichzeitig als Stellvertretender Direktor eingesetzt.

Zum 30.09. geht Prof. Sachtleben als Ordentliches Mitglied der Akademie und Institutsdirektor in den Ruhestand. Direktor wird am 1.10. Dr. Heinz Fankhänel, der aus dem Institut für Forstwissenschaften Eberswalde stammt. Er betreibt die Verlegung des DEI von Berlin nach Eberswalde.

Die Mitarbeiter der taxonomisch und bibliographisch arbeitenden Gruppen wenden sich gegen den Umzug mit dem Argument, dass Sammlungen und Bibliothek angesichts ihres internationalen Ranges nicht in eine Kreisstadt gehören. Für die Aufnahme des DEI wird das Rote Gebäude („Neue Forstakademie“ ), Schicklerstraße 5, der vormaligen, 1962 aufgelösten Forstwirtschaftlichen Fakultät der Humboldt-Universität zu Berlin umgebaut.

1963 Ab Sommer Umzug des DEI nach Eberswalde, Abschluss im November. Nach dem Umzug arbeiten 39 Personen am DEI. Die Verlegung verbessert die Raumsituation und die Arbeitsbedingungen wesentlich, während der Standortnachteil auch später kaum auszugleichen ist.

Die Abteilung für bibliographische Entomologie und die Schriftleitung der DEI-Zeitschriften übernimmt Dr. Günter Morge (1925-1984) in der Nachfolge von H. Sachtleben.

Der „Index Litteraturae Entomologicae, Serie II" erscheint in 4 Bänden mit insgesamt 2385 Seiten. Der Registerband V wird erst 1975 gedruckt.

Neu aufgenommen wird eine Reihe „Bibliographie der Bestimmungstabellen europäischer Insekten", beginnend mit dem Berichts-Jahr 1880, erstmalig erschienen 1967 (U. GöLLNERScheiding), danach fortgeführt von R. GAEdiKe (Beiträge zur Entomologic und Nova Supplementa Entomologia) bis zum Berichtsjahr 2000.

1968 Veröffentlichung des ersten Beitrages einer neuen Reihe: „Katalog der in den Sammlungen des Deutschen Entomologischen Instituts aufbewahrten Typen " in den Institutszeitschriften (Petersen \& Gaedike 1968). Bisher sind 26 Teile des "Typenkatalogs" erschienen.

1970 Das Präsidium der Akademie der Landwirtschaftswissenschaften der DDR (AdL) fasst im Rahmen einer "Akademiereform" Beschlüsse, mit denen das Deutsche Entomologische Institut seine Selbständigkeit und seinen traditionsreichen, weltweit renommierten Namen verliert. Prof. H. Fankhänel scheidet aus dem Amt. Die bereits komplett vorbereitete 11. Wanderversammlung Deutscher Entomologen in Halle (Saale) - die letzte - muss abgesagt werden.

In dieser politischen Phase werden in der DDR alle offiziellen Bezeichnungen, die eine gesamtdeutsche Geltung vermuten lassen könnten, auf „DDR“ umgestellt, so auch die „Deutsche Akademie der Wissenschaften" in "Akademie der Wissenschaften der DDR". (Am Namensbestandteil "Deutsches" des DEI im Sinne von "gesamtdeutsch" haben nach 1989 auch noch andere Gremien Anstoß genommen.) 
Die Max-Planck-Gesellschaft verkauft das ursprüngliche Institutsgebäude in Berlin-Dahlem, Gosslerstraße 20, an die Stadt Berlin zur dauerhaften Nutzung durch die Freie Universität.

1971 Das Deutsche Entomologische Institut wird zu einer Abteilung des neu gebildeten Instituts für Pflanzenschutzforschung Kleinmachnow (IPF, ehemals Biologische Zentralanstalt, BZA). Die Leitung der „Abteilung“ wird Dr. Günter Morge übertragen. Sie untersteht ihrerseits dem Leiter des IPF-Bereiches Eberswalde, Prof. Dr. Werner Ebert (geb. 1928), der selbst Entomologe ist und sich mit Überwachung und Prognose von Schaderregern beschäftigt.

Der neue Adressname „Akademie der Landwirtschaftswissenschaften, Institut für Pflanzenschutzforschung Kleinnachnow, Bereich Eberswalde, Abteilung Taxonomie der Insekten (ehemals DEI)" ist in der Tat monströs und namentlich im Ausland kaum vermittelbar. Dessen ungeachtet genießt das „ehem. DEI" unausgesprochen eine gewisse Autonomie und besondere Wertschätzung der Akademie-Leitung. Seine Arbeitsthemen, die zweifellos nicht im Fokus der landwirtschaftlichen Forschung liegen, werden gefördert, und internationale Verbindungen (Schriftentausch) können im Prinzip weiter gepflegt werden - für die AdL eine ungewöhnliche Situation. Auch die petsonelle Ausstattung ist gut (6 Wissenschaftler, 9 technische Mitarbeiter).

Am Haus verbleiben Forschungsaufgaben zur Systematik und Taxonomie der Insekten, die Sammlungsbetreuung, die bibliographischen Arbeiten sowie die entomologische Auskunftstätigkeit. Die vormalige DEI-Abteilung Insekten-Ökologie wird abgetrennt und einer anderen „Abteilung" des „Bereichs Eberswalde" angeschlossen, deren Leitung Prof. Ebert als Bereichsdirektor obliegt.

1984 Prof. Dr. G. Morge stirbt überraschend. Abteilungsleiter wird Dr. Günther Petersen. Die Entomologische Bibliothek wird abgetrennt und mit der Bereichs-Informationsstelle zu einem Informationszentrum vereinigt (Leiter: Dr. Klaus Rohlfien). Sammelschwerpunkt der Bibliothek bleibt aber das internationale entomologische Schriftum.

1985 Wiederbelebung der früheren Supplement-Reihe als „Nova Supplementa Entomologica“ durch Dr. Rohlfien mit einem 1. Band: „Periodica entomologica. Bestandsverzeichnis der laufend gehaltenen Zeitschriften und Serien [der Entomologischen Bibliothek]“ (Wutzmer et al. 1985).

Die Entomologische Bibliothek besitzt 21000 Einzelwerke, 40000 Bände Zeitschriften und Serien, 103000 Sonderdrucke, 10000 Kleinschriften, 1000 Mikrofilme sowie 500 Karten. Die Sammlung besteht aus über 2 Millionen Exemplaren von ca. 275000 Arten.

1986 Das einhundertjährige Bestehen des Deutschen Entomologischen Instituts wird zumindest literarisch ausführlich gewürdigt (EBERT et al. 1986). Zur Jubiläumsfeier im Hörsaal des Instituts (bzw. der Abteilung!) sind nur wenige Gäste geladen, darunter der Zentrale Fachausschuss Entomologie des Kulturbundes der DDR. Die sehr inhaltsreiche Festrede hält Dr. Petersen (Petersen 1986).

1989 Die Leitung der Abteilung Taxonomie der Insekten geht nach der Berentung von Dr. G. Petersen im September an Dr. Reinhard Gaedike (geb. 1942) über.

Wendezeit auch im Bereich Eberswalde. Es wird klar, dass nach der deutschen Einigung die Biologische Bundesanstalt für ganz Deutschland zuständig sein wird und das IPF Kleinmachnow so nicht bestehen bleiben kann. Die Mitarbeiter des DEI bemühen sich frühzeitig um die ReEtablierung des Instituts und werden darin vom Bereichsleiter W. Ebert unterstützt. 
1990 Das Institut für Pflanzenschutzforschung Kleinmachnow der AdL wird aufgelöst; gleichzeitig wird die Biologische Zentralanstalt Berlin mit Sitz in Kleinmachnow in anderem Arrangement (wieder)gegründet. In der Gründungsanweisung findet sich auf Initiative der DEI-Mitarbeiter auch das Deutsche Entomologische Institut unter seinem alten Namen. Im Januar übernimmt Prof. Ebert die „Abteilung“, die sich ab Juli als Institut formiert. Die entomologische Bibliothek wird aus der ehemaligen BereichsInformationsstelle herausgelöst und wieder mit dem DEI vereinigt. W. Ebert leitet das DEI bis zu seinem Eintritt in den Vorruhestand (30.09.), danach (zum 1.10.) wählt die Belegschaft Dr. Joachim Oehlke zum Geschäftsführenden Direktor.

Der Direktor des Forschungsinstitutes und Museums Alexander Koenig (Bonn), Prof. Dr. Clas M. Naumann, setzt sich bei der Brandenburger Landestegierung, bei Bundesministerien und dem Wissenschaftsrat wiederholt für den Erhalt des DEI ein. Zwischen DEI und dem Museum Koenig wird ein Partnerschaftsabkommen geschlossen (28.11.).

1991 Der Wissenschaftsrat evaluiert die Eberswalder Institute und empfiehlt die weitere Förderung des DEI in Verbindung mit dem Museum für Naturkunde Berlin. Details soll eine spätere gemeinsame Evaluierung festlegen.

Begehung am 12.02. Vorsitzender der Kommission ist Prof. Dr. Gerhard Neuweiler (München). Empfehlung des Wissenschaftsrats (27.09.):- Deutsches Entomologisches Institut: dieses Institut verfügt über eine bedeutende Insektensammlung und Fachbibliothek und ist auf dem Gebiet der Taxonomie führend. Deshalb gilt es, diese Einrichtung zu erhalten und das dort vorhandene Insektenmaterial mittels moderner biochemischer Methoden für Forschungszweckezu erschließen. Es wird empfohlen, das DEI als weiteres selbständiges Institut dem Naturkundemuseum Berlin anzugliedern und für diese Einrichtung eine Personalstärke von 8 Wissenschaftlern und 8 technischen Angestellten vorzusehen. Der Standort Eberswalde sollte beibehalten werden. Wegen dem erneuerungsbedürftigen Gebäudebestand des Naturkundemuseums würde es sich anbieten, die dort vorhandene entomologische Sammlung nach Eberswalde zu verlagern.

1992 Trotz der positiven Einschätzung entsteht eine schwierige Lage, weil die Brandenburger Regierung nun eine Zuständigkeit Berlins sieht, der Berliner Senat sich aber auf das „Belegenheitsprinzip“ beruft. J. Oehlke erreicht mit großem Einsatz eine interimistische Förderung des DEI als „Projektgruppe Entomologie“ im Wissenschaftler-Integrationsprogramm (WIP, koordiniert durch KAI e. V., Berlin). Weitere Mittelzuwendungen - darunter die Bibliotheksförderung - erfolgen aus Projektanträgen an Brandenburger Ministerien. Die Deutsche Forschungsgemeinschaft nimmt die B15 schon 1991 in ihr Programm für Spezialbibliotheken auf.

Zum 30.06. endet die Trägerschaft durch das Landwirtschafts-Ministeriums von Brandenburg. Das Wissenschafts-Ministerium bestimmt, dass der Name „Deutsches Entomologisches Institut “ nicht verwendet werden darf, da keine institutionelle Förderung vorliegt. Aus Bundes- und Landesmitteln finanzieren sich umfangreiche Instandsetzungs- und Sicherungsarbeiten am denkmalgeschützten Gebäude. Eine öffentliche Insekten-Ausstellung mit lebenden Insekten (rund 60 Arten) wird eingerichtet; Titel: „Insekten - Faszinationen in Natur, Sammlung und Buchkunst".

1993 Zur Überbrückung wird eine vorübergehende Integration der Projektgruppe Entomologie in die neu gegründete Fachhochschule Eberswalde beantragt. J. Oehlke erhält einen Ruf als Professor für Tierökologie an die Fachhochschule und verlässt das DEI. Auf Empfehlung Oehlkes übernimmt Prof. Dr. Holger H. Dathe am Jahresende die Leitung. 
H. H. Dathe (geb. 1945) wird auf Antrag des Brandenburger Wissenschafts-Ministeriums von seiner Dienststelle, dem Institut für Zoo- und Wildtierforschung in Berlin, für die Leitung des DEI in der neuen Evaluierungsphase abgeordnet. Nach kurzer Einarbeitung beantragt er einen Aufhebungsvertrag und bindet sich zum 1.01.1994 fest an das Institut. - Dathe hat 1972 über ein Thema zum akustischen Verhalten von Insekten promoviert (Mentor Prof. Dr. G. Tembrock, Humboldt-Universität zu Berlin) und eine Reihe hymenopterologischer Arbeiten publiziert, meist in enger Verbindung mit dem Zoologischen Museum Berlin (Dr. E. Königsmann) und dem Deutschen Entomologischen Institut (Dr. J. Oehlke).

Gründung des Vereins der Freunde und Förderer des Deutschen Entomologischen Instituts e. V. (23.09.), ausdrücklich Bezug nehmend auf einschlägige Traditionen des DEI. Im Vereinstegister seit dem 16.11. unter 3VR335 beim Registergericht Eberswalde.

1994 Integration der „Projektgruppe Entomologie“ in die Fachhochschule Eberswalde (1.01.). Für die Weiterförderung werden 3 Projekte beim Land beantragt, außerdem mehrere Arbeitsbeschaffungsmaßnahmen (ABM), darunter eine für die Insekten-Ausstellung.

Projekt 1. Wissenschaftliche Untersuchungen zur Ermittlung repräsentativer Gliedertierarten bzw. -gruppen für ausgewählte Ökosystemtypen des Landes Brandenburg (1 wissenschaftlicher Mitarbeiter, 2 wissenschaftlich-technische Mitarbeiter);

Projekt 2. Inhaltliche Formal- und Sacherschließung sowie Bestandsführung der Entomologischen Bibliothek (1 Bibliotheksleiterin, 1 Bibliothekarin, 2 Bibliotheksmitarbeiterinnen, 1 Hilfskraft);

Projekt 3. Quantitative und qualitative Erfassung von Gliedertieren in ausgewählten Gebieten des Landes Brandenburg (1 wissenschaftlicher Mitarbeiter, 4 wissenschaftlich-technische Mitarbeiter).

1995 Erneute Evaluierung durch den Wissenschaftstat, gemeinsam mit dem Museum für Naturkunde Berlin; Begehung des DEI am 9.02. Vorsitzender der Gruppe ist Prof. Dr. Gotthilf Hempel (Bremerhaven).

Mündlich mitgeteiltes Ergebnis (lt. Schreiben von H. H. Dathe an die Partner des DEI):

(1) Das DEI wird in das Museum für Naturkunde Berlin (MAN) eingegliedert und mit diesem zusammen in die Blaue Liste aufgenommen werden.

(2) Die Berliner Entomologie und das DEI werden zu einem gemeinsamen (vierten) Institut des MfN verbunden, das den Namen „Deutsches Entomologisches Institut“ trägt.

(3) Der Standort Eberswalde wird erhalten, wobei hier die ökologische Entomologie bestimmend wird. In Berlin bilden Taxonomie und Systematik die Schwerpunkte, entsprechend werden dort die Sammlungen konzentriert.

(4) Für die Leitung des neuen DEI ist - entsprechend den Blaue-Liste-Gepflogenheiten und in Gleichrangigkeit zu den drei anderen Instituten des MfN - eine C4-Stelle vorzusehen. Die Zuständigkeit dafür wird beim Land Brandenburg gesehen.

(5) In der Zwischenperiode bis zur Bund/Länder-Finanzierung sollte das Land Brandenburg in Abstimmung mit dem Bund eine Überbrückung für das DEI schaffen.

Der Wissenschaftsrat stellt ergänzende Fragen (14.07.) und fordert schließlich in einem weiteren Termin mit der Evaluierungsgruppe am 13.11. ein gemeinsames Konzept von Naturkundemuseum Berlin und "Projektgruppe Entomologie“ Eberswalde, bevor eine Empfehlung des Wissenschaftsrats ausgesprochen werden könne.

1996 Das gemeinsame Konzept wird termingemäß im geforderten Sinne eingereicht (4.01.). In der schriftlichen Stellungnahme vom 12.07. empfiehlt der Wissenschaftsrat, das MfN und die Projektgruppe Entomologie institutionell zusammenzuführen. 
Zu diesem Zweck soll im MfN neben den drei bestehenden Instituten ein Institut für systematische und ökologische Entomologie als vierte wissenschaftliche Einheit geschaffen werden, die den Namen „Deutsches Entomologisches Institut" tragen soll und das vorhandene entomologische Potential des MfN in Berlin und der Projektgruppe Entomologie, Eberswalde, zusammenfasst. Für den Leiter des Deutschen Entomologischen Instituts wird die Einrichtung einer C4Stelle an der Humboldt-Universität empfohlen. Zusätzlich zum Leiter sind an der bisherigen Projektgruppe Entomologie je 8 Stellen wissenschaftliche und nichtwissenschaftliche Mitarbeiter sowie drei Stellen für Bibliothekskräfte erforderlich. Der Standort Eberswalde soll als Teilstandort des neuen Instituts bestehen bleiben. Mittel- und längerfristig ist die systematische Entomologie in Berlin, der ökologische arbeitende Teil in Eberswalde zu konzentrieren. Das Konzept des neuen Entomologischen Instituts soll von einem von den Ländern Berlin und Brandenburg einzurichtenden Gründungskomitee entwickelt werden, das aus externen Experten besteht.

Allerdings ist das Museum für Naturkunde Berlin nicht ernsthaft bereit, die Empfehlungen umzusetzen, insbesondere bezüglich der Empfehlung, die Entomologie aus dem Institut für Systematische Zoologie herauszulösen und als Deutsches Entomologisches Institut mit den Eberswaldern $\mathrm{zu}$ vereinigen (Schreiben an den 1. Vizepräsidenten der HUB). Zudem ist eine Aufnahme des Museums in die Blaue Liste (Leibniz-Gemeinschaft, WGL) nicht in Sicht, so dass das DEI ungeachtet aller Empfehlungen im Land Brandenburg auf sich gestellt bleibt.

1997 Vereinsgründung des Deutschen Entomologischen Instituts e.V. (23.06.), gleichzeitig Herauslösung aus der Fachhochschule Eberswalde. Ein weiterer Verbleib in der Fachhochschule hätte arbeitsrechtlich zur - aus Sicht des Landes Brandenburg - unerwünschten Festanstellung der DEI-Mitarbeiter und zur Institutionalisierung des DEI geführt.

Gründungsmitglieder: Prof. Dr. Erich Dickler (Deutsche Gesellschaft für allgemeine und angewandte Entomologiee.V.), Prof. Dr. Holger H. Dathe, Dr. Reinhard Gaedike (beide Deutsches Entomologisches Institut), Prof. Dr. Hannelore Hoch (Humboldt-Universität zu Berlin), Josef Keil (Vorstand der Sparkasse Barnim), Prof. Dr. Bernhard Klausnitzer (Entomofaunistische Gesellschaft e.V.), MR Dr. Ursula Kleinhans (Ministerium für Wissenschaft, Forschung und Kultur des Landes Brandenburg), Prof. Dr. W. Laux (AG der Spezialbibliotheken e.V. im Deutschen Bibliotheksverband), Dr. Siegfried Löser (Entomologische Gesellschaft Düsseldorf), Prof. Dr. Gerd Müller-Motzfeld (Bundesfachausschuss Entomologie, Naturschutzbund Deutschland/ Universität Greifswald), Prof. Dr. Clas M. Naumann (Zoologisches Forschungsinstitut und Museum Alexander Koenig, Bonn), Prof. Dr. Joachim Oehlke (Verein der Freunde und Förderer des DEI), Dr. Günther Petersen, Reinhard Schulz (Bürgermeister der Stadt Eberswalde), Prof. Dr. Gunther Wolff (Fachhochschule Eberswalde). - Prof. Dathe wird zum Vorstand gewählt, Dr. Gaedike zu seinem Vertreter. Wissenschaftlicher Beirat: Prof. Hoch, Prof. Müller-Motzfeld, Prof. Naumann, Prof. Dr. Rainer Willmann (Universität Göttingen). Der Verein fungiert als Träger des Forschungsinstitutes.

1999 5.11. Beschluss zur Selbstauflösung des DEI-Vereins unter der Maßgabe einer Integration ab 1.01. in das Zentrum für Agrarlandschafts- und Landnutzungsforschung (ZALF) e.V. Müncheberg. Die Landwirtschaftsressorts des Bundes und des Landes stellen für die Institutionalisierung des DEI rund 2 Millionen DM bereit.

Spätestens zum Jahresende 2000 hätte durch das allgemeine Ende des Wissenschaftler-IntegrationsProgramms kein Bundesanteil mehr zur Finanzierung des DEI zur Verfügung gestanden; eine reine Landes-Förderung wird ausgeschlossen. Als Lösung, der sich der Wissenschaftsrat wie auch die Mitglieder des DEI e. V. nur zögernd anschließen, beantragt das Brandenburger Wissenschaftsministerium - in Übereinkunft mit den beteiligten Leitern - die Integration des DEI in das ZALF. (DATHE 2000) 
2000 Zum 1.01. wird das DEI als 7. Institut in das ZALF eingegliedert. Dabei werden 20 Mitarbeiter des DEI auf Dauerstellen übernommen. Die Ausstellung mit Insektarium im Eberswalder Institutsgebäude wird zur Überbrückung vom Brandenburger Wissenschaftsministerium weiter gefördert.

2001 Die Ausstellung kann vom Wissenschaftsministerium nicht dauerhaft gefördert werden; ein anderer (kommunaler) Träger findet sich trotz Bemühung einiger Stadtverordneter in Eberswalde nicht. Sie wird im August geschlossen.

2004 Fertigstellung der Baumaßnahmen am neuen Institutsgebäude in Müncheberg und Umzug des DEI. (Dathe 2005)

2005 Externe Evaluierung des ZALF durch die Leibniz-Gemeinschaft. Der Vorsitzende, Prof. Dr. Dietrich Wegener (Dortmund), bekundet nach der Begehung am 25.05. die vorläufige Intention der Evaluierungskommission, das DEI zum Anschluss an das Forschungsinstitut und Naturmuseum Senckenberg in Frankfurt am Main vorzuschlagen. Damit soll eine bessere fachliche Einordnung des DEI innerhalb der WGL erreicht werden.

\section{Danksagung}

Bei der Vorbereitung und Durchsicht des Manuskriptes unterstützten mich zahlreiche Kollegen, denen ich zu Dank verpflichtet bin. Herausheben möchte ich die Mitwirkung von Dr. Reinhard Gaedike (Bonn), Prof. Dr. Bernhard Klausnitzer (Dresden), Dr. Gerd Lutze (Eberswalde), Dr. Günther Petersen (Tauche) und Jutta Valentin-Dockendorf (Müncheberg). Vor allem konnte ich mich auf die umfangreichen, langiährig sorgfältig geführten Unterlagen von Dr. Klaus Rohlfien (Eberswalde) stützen, wofür ich herzlich danke.

\section{Literatur}

DAL 1953-1969: Jahrbuch. Deutsche Akademie der Landwirtschaftswissenschaften zu Berlin. 1, 1952 (1953) - 1967/1968 (1969). - Berlin: Akademie-Verlag.

Dathe, H. H. \& Rohlfien, K. 1994: Das Deutsche Entomologische Institut in Eberswalde. - hochschule ost, Leipzig 4 (5/94): 68-81.

Dathe, H. H. 1995: Das Deutsche Entomologische Institut in der Waldstadt. - Eberswalder Jahrbuch für Heimat-, Kultur- und Naturgeschichte 1995/1996, Eberswalde: 56-64.

Dathe, H. H. 1999: Deutsches Entomologisches Institut e. V. - Mitteilungen des Internationalen Entomologischen Vereins, Frankfurt a. M. 24 (1/2): 84-87.

Dathe, H. H. 2000: Aufgaben des Deutschen Entomologischen Instituts. Arbeitsergebnisse des Jahres 1999. Chronik. - In: Jahresbericht 1999 „Deutsches Entomologisches Institut", Eberswalde: 4-9.

Dathe, H. H. 2004: Ein Blatt des Gedenkens an Clas M. Naumann (1939-2004). - Beiträge zur Entomologie, Keltern 54 (1): 1-2.

Dathe, H. H. 2005: Das neue Forschungsgebäude des Deutschen Entomologischen Instituts am ZALF in Müncheberg. - DGaaE Nachrichten, Darmstadt 19 (1): 52-56.

Dathe, H. H. 2005: Neues Forschungsgebäude für das DEI in Müncheberg, - Entomologische Nachrichten und Berichte, Dresden 49 (1): 44.

Ebert, W.; Rohlpien, K.; Petersen, G. \& Friese, G. 1986: Einhundert Jahre Deutsches Entomologisches Institut. - Beiträge zur Entomologie, Berlin 36 (1): 5-52. - Hier Angaben zum Großteil weiterer Originalquellen. Als pdf-Datei erhältlich auf http:/www.zalf.de/home_zalf/download/dei/100JahreDEI. pdf. 
FANKHÄNel, H. 1966: 80 Jahre Deutsches Entomologisches Institut. - Beiträge zur Entomologie, Berlin 16: 259-269.

Fankhänel, H. \& Rohlfien, K. 1969: Das Deutsche Entomologische Institut in Eberswalde der Deutschen Akademie der Landwirtschaftswissenschaften zu Berlin. - Entomologische Berichte, Berlin 2/ 1969: 61-66.

Fruese, G. \& Königsmann, E. 1962: Ergebnisse der Albanien-Expedition 1961 des Deutschen Entomologischen Institutes. 1. Beitrag. Bericht über den Verlauf der Reise. - Beiträge zur Entomologie, Berlin 12 (7/8): 765-843, 8 Taf., 9 Karten.

GAEDIKE, R. 1995: Collectiones entomologicae (1961-1994). - Nova Supplementa Entomologica, Berlin 6: $1-84$.

GAEDIKE, R. 1995: Deutsches Entomologisches Institut: origin, history and present. - Holarctic Lepidoptera, Gainesville 282: 51-56.

GAEDIKE, R. 1996: Bibliothek des Deutschen Entomologischen Instituts. - In: KRAuse, F. (Hrsg.) Handbuch der historischen Buchbestände in Deutschland. - Hildesheim, Zürich, New York: Olms-Weidmann 16: 311-314.

Gafdike, R. \& Smetana, O. 1978: Ergänzungen und Berichtigungen zu Walther Horn und Sigmund Schenkling: Index Litteraturae Entomologicae. Serie I: Die Welt-Literatur über die gesamte Entomologie bis inklusive 1863. Teil I: A-K. - Beiträge zur Entomologie, Berlin 28 (2): 329-436.

Gaedike, R. \& Smetana, O. 1984: Dass., Teil II; L-Z. - Beiträge zur Entomologie, Betlin 34 (1): 167-291.

GöllnER-Scheiding, U. 1967: Bibliographie der Bestimmungstabellen europäischer Insekten (18801963). Teil I: Apterygota bis Siphonaptera, - Beiträge zur Entomologie, Berlin 17 (5/8): 697-958.

Hennig, W. 1950: Grundzüge einer Theorie der Phylogenetischen Systematik. - Berlin: Deutscher Zentralverlag: $370 \mathrm{~S}$.

Horn, W. 1912: Skizzen aus der Geschichte des „Deutschen Entomologischen Museums“. - Entomologische Mitteilungen, Berlin 1: 1-6, 33-38.

Horn, W. 1922: Et meminisse et vaticinari liceat. II. Über Zola's „J'accuse .....". - Entomologische Mitteilungen, Berlin 11 (5): 199-208.

Horn, W. 1936: Das Deutsche Entomologische Institut der Kaiser-Wilhelm-Gesellschaft in Berlin-Dahlem. - In: 25 Jahre Kaiser-Wilhelm-Gesellschaft zur Förderung der Wissenschaften. Bd. 1: 99-102. - Berlin.

HoRn, W. \& KaHLE, I. 1935-1937: Über entomologische Sammlungen, Entomologie und Entomo-Museologie. - Entomologische Beihefte, Berlin 2 (1935): 1-160. 3 (1936): 161-296. 4 (1937): 297-536 + 38 Taf.

Horn, W.; Kahle, I.; Friese, G. \& Gaedike, R. 1990: Collectiones entomologicae. Ein Kompendium über den Verbleib entomologischer Sammlungen der Welt bis 1960. Teil I, II. - Berlin: Akademie der Landwirtschaftswissenschaften der DDR: 573 S. (Addenda et corrigenda, s. Gaedike, R. 1995).

Horn, W. \& Schenkung, W. 1928/1929: Index Litteraturae Entomologicae. Serie I: Die Welt-Literatur über die gesamte Entomologie bis inklusive 1863. Band I-IV. - Berlin: $1426 \mathrm{~S}$.

KNORre, D. von 2004: Warum und zu welchem Zweck pflegen und erhalten wir naturkundliche Sammlungen? - Mainzer naturwissenschaftliches Archiv 42: 302-313.

Köppen, H. 1967: Gustav Kraatz - Forscher, Schöpfer, Mensch -, ein Beitrag zur Geschichte seines Lebens. - Beiträge zur Entomologie, Berlin 17 (3/4): 375-387.

KorschefsKx, R. 1936: Zum fünfzigjährigen Bestehen des Deutschen Entomologischen Institutes der Kaiser-Wilhelm-Gesellschaft, Berlin-Dahlem. - Entomologische Blätter, Berlin 32: 89-93.

KraATZ, G. 1870: Vereinsangelegenheiten. - Betliner Entomologische Zeitschrift, Berlin 14: XI.

KRAATZ, G. 1876: Über ein Deutsches Entomologisches National-Museum. - Entomologische Monatsblätter, Berlin 1: 6-9.

KraATZ, G. 1880: Das Deutsche Entomologische Nationalmuseum und die bisherigen Erklärungen zu Gunsten desselben. - Deutsche Entomologische Zeitschrift, Berlin 1880: 9-14. 
KraAtz, G.; Heyden, L. von.; Koltze, W.; Roeschke, H. \& Horn, W. 1904: Das Deutsche Entomologische National-Museum. - Deutsche Entomologische Zeitschrift, Berlin 1904: 461-463.

Kroel, D. \& RohlfieN, K. 1995: Deutsches Entomologisches Institut. Kleiner Institutsführer. - Eberswalde: Deutsches Entomologisches Institut: $100 \mathrm{~S}$.

MaRwinski, F. \& WUtzmer, C. 1973: Aus der Geschichte der Bibliothek des ehemaligen Deutschen Entomologischen Institutes. - Beiträge zur Entomologie, Berlin 23: 197-217.

MARWINSKI, F. 1970-76: Nachlässe und Konvolute. - Beiträge zur Entomologie 20: 813-826; 21: 161-164; 205-212; 23: 427-443; 24: 349-382; 25: 163-188; 297-323; 26: 167-178.

Petersen, G. \& Gaedike, H. 1968: Katalog der in den Sammlungen des Deutschen Entomologischen Institutes aufbewahrten Typen - I (Ephemeroptera, Odonata, Plecoptera). - Beiträge zur Entomologie, Berlin 18 (7/8): 959-969.

Petersen, G. 1986: Rede zut 100-Jahr-Feier des Deutschen Entomologischen Institutes im Institut. Manuskript, DEI-Archiv: $25 \mathrm{~S}$.

RoHLFien, K. 1996: Entwicklungsetappen des Deutschen Entomologischen Instituts. - Eberswalder Jahrbuch für Heimat-, Natur- und Kulturgeschichte 1996/1997. - Verein für Heimatkunde zu Eberswalde e.V., Eberswalde: 216-226.

SACHTLEBEN, H. 1936: 50 Jahre Deutsches Entomologisches Institut der Kaiser-Wilhelm-Gesellschaft. - Arbeiten über morphologische und taxonomische Entomologie, Berlin 3: 169-172.

SACHTLEBEN, H. 1962: 75 Jahre Deutsches Entomologisches Institut. - In: Deutsche Akademie der Landwirtschaftswissenschaften zu Berlin, Tagungsberichte, Nr. 45. - Bericht über die 9. Wanderversammlung Deutscher Entomologen, 6. bis 8. Juni 1961 in Berlin. - Berlin: 13-28.

SCHWARTz, M. 1934: Dahlemer Arbeitsgemeinschaft. - Arbeiten über morphologische und taxonomische Entomologie, Berlin 1: 1-6.

TAeger, A. \& Dathe, H. H. 2005: Das Deutsche Entomologische Institut - Umzug nach Eberswalde. - Museum aktuell, München N.. 121, Dez. 2005/Jan. 2006: 28-30.

WagemanN, H. \& Dathe, H. H. 2006: Deutsches Entomologisches Institut Berlin-Dahlem / BerlinFriedrichshagen / Eberswalde / Müncheberg. - Band I/2, S. 74-93. - In: WAGEMANN, H. (Hrsg.): Von der Deutschen Akademie der Landwirtschaftswissenschaften zu Berlin zur Akademie der Landwirtschaftswissenschaften der DDR. Ein Beitrag zur Geschichte 1951-1991. - Berlin: Verlag am Park.

Wutzmer, C.; Marwinski, F. \& Fritz, D. 1985: Periodica entomologica. Bestandsverzeichnis der laufend gehaltenen Zeitschriften und Serien. - Eberswalde-Finow: Akademie der Landwirtschaftswissenschaften der DDR, Institut für Pflanzenschutzforschung Kleinmachnow, Entomologische Bibliothek: 235 S.

\author{
Anschrift des Verfassers: \\ Prof. Dr. Holger H. Dathe \\ Deutsches Entomologisches Institut \\ Leibniz-Zentrum für Agrarlandschaftsforschung (ZALF) \\ Eberswalder Straße 84 \\ D-15374 Müncheberg \\ dathe@zalf.de
}

